\title{
XMM-Newton observations of the two X-ray weak quasars PG 1411+442 and Mrk 304
}

\author{
W. Brinkmann ${ }^{1}$, I. E. Papadakis ${ }^{3,4}$, and E. Ferrero ${ }^{2}$ \\ ${ }^{1}$ Centre for Interdisciplinary Plasma Science, Max-Planck-Institut für extraterrestrische Physik, Postfach 1312, \\ 85741 Garching, Germany \\ 2 Max-Planck-Institut für extraterrestrische Physik, Postfach 1312, 85741 Garching, Germany \\ 3 Physics Department, University of Crete, 71003 Heraklion, Crete, Greece \\ 4 IESL, FORTH-Hellas, 71 110, Heraklion, Crete, Greece
}

Received 29 July 2003 / Accepted 14 October 2003

\begin{abstract}
We present the results of XMM-Newton observations of two X-ray weak quasars, PG 1411+442 and Mrk 304. In both cases the spectra can basically be represented by hard power laws with photon indices typical for radio-quiet quasars, heavily intrinsically absorbed by column densities of $\sim 2 \times 10^{23} \mathrm{~cm}^{-2}$ in PG $1411+442$ and $\sim 4 \times 10^{22} \mathrm{~cm}^{-2}$ in Mrk 304. The data require in both cases an additional steep $(\Gamma \sim 3)$ soft power law component, absorbed only by the galactic column density towards the quasars. The soft parts of the spectra exhibit additional complex structures like emission line features around $\sim 0.59 \mathrm{keV}$, and absorption edges around $0.7 \mathrm{keV}$ and $\sim 1 \mathrm{keV}$. These features strongly suggest the presence of "warm" (i.e. ionized) material in the central region of the two sources. Due to the insufficient photon statistics and to the lack of more advanced models for the spectral fitting, we are unable to determine accurately the geometry and physical state of the absorbing material. The physical implications of various viable spectral absorption or scattering scenarios for the nature of these objects and of the X-ray weak quasars in general, are discussed.
\end{abstract}

Key words. galaxies: active - quasars: general - quasars: individual: PG 1411+442, Mrk 304

\section{Introduction}

The broad band spectral energy distribution (SED) of quasars provides important information about the physical conditions governing the emission of radiation and can thus be used for a phenomenological classification of the quasars. For example, the ratio of the flux emitted in the radio to the optical band separates radio-loud and radio-quiet quasars; both classes are obviously differing dramatically in their emission properties. Another characteristic quantity is the the broad band spectral index from the ultra-violet $(2500 \AA)$ to the X-ray $(2 \mathrm{keV})$ band, which is defined as the luminosity ratio $\alpha_{\mathrm{ox}}=$ $-0.384 \log \left(l_{2 \mathrm{keV}} / l_{2500} \AA\right)$, the so called X-ray loudness. For the large sample of radio-quiet quasars studied by ROSAT (Yuan et al. 1998a) the average value is $\sim 1.6$ with a dispersion of $\sigma=0.19$ (for $0.2 \leq z \leq 0.5$ ). A more detailed analysis showed that the apparent redshift dependence of $\alpha_{\mathrm{ox}}$ can emerge in a sample with an intrinsic luminosity independent $\alpha_{\text {ox }}$ provided that dispersion of the optical luminosities deviating from the average SED are similar or larger than that of the X-ray luminosities (Yuan et al. 1998a; Yuan et al. $1998 \mathrm{~b}$ ). Thus the X-ray loudness $\alpha_{\mathrm{ox}}$ is very likely an evolution and luminosity independent, class specific quantity of quasars,

Send offprint requests to: $\mathrm{W}$. Brinkmann,

e-mail: wpb@mpe.mpg.de although there are recent claims (Vignali et al. 2003) that in a sample of radio-quiet quasars from the Sloan Digital Sky Survey $\alpha_{\text {ox }}$ steepens at high UV luminosities.

Only six out of the 377 sources detected by ROSAT had an $\alpha_{\text {ox }}>2$, i.e., an X-ray flux by a factor of $\sim 30$ lower at the same optical flux than the bulk of the sample. These objects do not include Broad Absorption Line (BAL) quasars, which remain undetected at all in the soft ROSAT All Sky Survey (Brinkmann et al. 1999). One of the $\alpha_{\mathrm{ox}}>2$ quasars, PG $0844+349$, turned out to be highly variable and therefore to be $\mathrm{X}$-ray quiet only in its low state (Wang et al. 2000; Brinkmann et al. 2003, and references therein). Thus, strong variability might be one reason for a quasar to be X-ray weak at certain times. From a Chandra "mini-survey" of 18 quasars, known to be X-ray weak from the ROSAT survey, Risaliti et al. (2003) suggest that most of these objects were intrinsically under-luminous and that they represent a quasar population with a SED different from that of standard blue quasars. On the other hand Brandt et al. (2000) find a strong correlation between $\alpha_{\mathrm{ox}}$ and the $\mathrm{C}_{\mathrm{IV}}$ absorption equivalent width in the Boroson \& Green (1992) sample of nearby bright quasars and conclude that absorption is the primary cause of the X-ray weakness in these objects. As there is the strong claim (Mathur 1994; Mathur et al. 1994) that the UV absorption is directly linked to the warm absorption found in many AGN a study of X-ray weak quasars can provide 
powerful constraints on the ionization structure of the absorber and the matter distribution in the sources.

That intrinsic absorption can play an important role in X-ray weak quasars was convincingly demonstrated in the analysis of an ASCA observation of PG 1411+442, a nearby $(z=0.0896)$ very bright $\left(m_{v}=14.99\right)$ quasar with prominent UV absorption. Classified as a BAL quasar by Malkan et al. (1987) PG 1411+442 shows line widths narrower than more typical BAL QSOs. From a ROSAT PSPC observation Rachen et al. (1996) found a steep power law photon index of $\Gamma=3.19 \pm 0.20$ and the fitted absorption was consistent with the galactic value $\left(N_{\mathrm{H}}=1.05 \times 10^{20} \mathrm{~cm}^{-2}\right.$, Lockman \& Savage 1995). The apparent X-ray loudness deduced from this measurement is $\alpha_{\mathrm{ox}} \sim 2.31$, however, the unusually steep X-ray slope introduces a considerable uncertainty is this determination. In the medium energy ASCA band the spectral slope appeared to be much flatter (Brinkmann et al. 1999). However, the spectrum could be well fitted by a combination of a steep soft power law $(\Gamma=3.07 \pm 0.12)$, with galactic absorption and a second, hard power law, with large intrinsic absorption of $N_{\mathrm{H}}=(2.4 \pm 0.5) \times 10^{23} \mathrm{~cm}^{-2}$. Due to the low photon statistics the slope of the hard power law had to be fixed arbitrarily at a value of $\Gamma=2.0$ and the data do not allow to distinguish whether the soft flux is scattered emission or the result of some kind of partial covering.

By comparing the normalizations at $1 \mathrm{keV}$ Brinkmann et al. (1999) find that the unabsorbed X-rays occur at the 3-5\% level of the intrinsic flux. As this is consistent with the fraction of the residual flux in the bottom of the UV absorption line troughs Wang et al. (1999) claim that the broad absorption lines are completely saturated, only scattered light is seen at the bottom of the lines.

The second object discussed in this paper is Mrk 304 (PG 2214+139), a bright $\left(m_{v}=15.08\right)$ nearby $(z=0.06576)$ optically quite well studied quasar. It shows weak radio emission but is, as PG $1411+442$, formally radio-quiet. First detected in X-rays by UHURU (Tananbaum et al. 1978) and later studied by Einstein (Tananbaum et al. 1986) it is a lowluminous object with rather uncertain spectral properties. In a ROSAT pointed PSPC observation the very weak source showed an unusually flat spectrum $(\Gamma=-0.07 \pm 0.94$, Rachen et al. 1996), again with large uncertainties, and it is listed by Wang et al. (1996) as a quasar with strong FeII emission.

In this paper we present an analysis of the XMM-Newton observations of PG $1411+442$ and Mrk 304. The next section starts with the observational details and a temporal analysis of the data. Section 3 deals with the spectral analysis of both sources, followed by a discussion of the physical implications of the results. Finally, in Sect. 5 we give a summary of the main conclusions.

\section{The data}

PG $1411+442$ was observed by XMM-Newton on July 10, 2002. The EPIC PN camera was operated in Extended Full Frame mode for a total of $\sim 27.5 \mathrm{ksec}$ with a thin filter (for details of the XMM instruments see Ehle et al. 2001). The EPIC MOS1 and MOS2 cameras were operated in Full

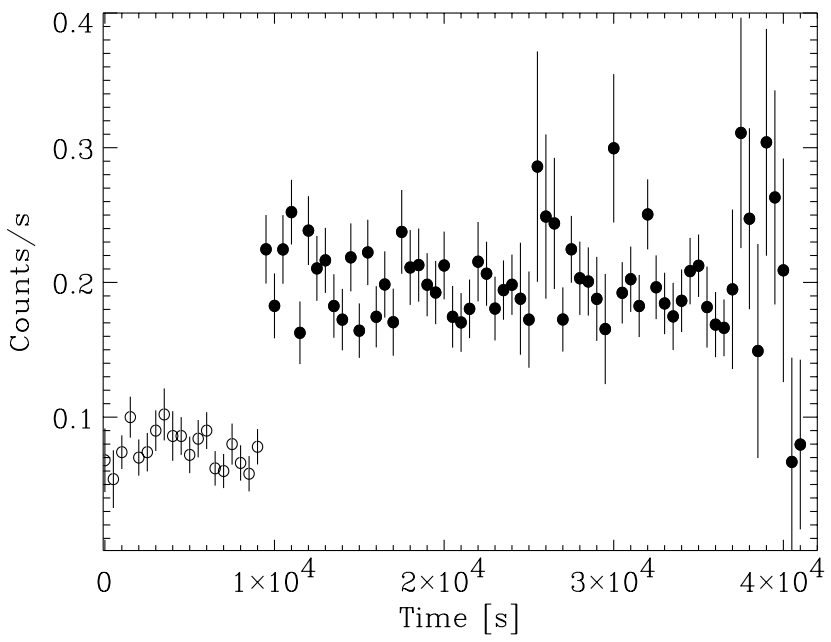

Fig. 1. Combined PN + MOS, background subtracted, 0.2-10 keV light curve of PG 1411+442; the time binning is $500 \mathrm{~s}$. The open symbols represent the MOS data only. The time axis starts at the beginning of the MOS exposure.

Window mode, with a total exposure of $\sim 41 \mathrm{ksec}$ each and with a thin filter. The two RGS were used in the Spectroscopy High Event Rate mode for $\sim 25 \mathrm{ksec}$ and the OM was operated in Imaging mode with the UVW2 filter.

The Mrk 304 observation took place on May 12, 2002. The PN observation had an effective exposure of $\sim 29 \mathrm{ksec}$, and the detector was operated in Extended Full Frame mode with a thin filter. The two MOS cameras were operated in Full Window mode with thin filter for about $35.7 \mathrm{ksec}$ each, and the RGS were used in Spectroscopy High Event Rate mode for $\sim 39 \mathrm{ksec}$ each.

All PN and MOS data were reprocessed using XMMSAS version 5.3.0; for the RGS data XMMSAS version 5.3.3 has been used. For the PN spectral analysis the latest response matrices (version 6.3, Dec. 2002) were taken. For the MOS cameras response matrices were created using the XMMSAS tool rmsgen. Unfortunately, in both observations the count rates in the RGS were too low for a meaningful spectral analysis.

For both PG $1411+442$ and Mrk 304, in the case of the MOS cameras we extracted the photons from a circular region centered on the source with a radius of $50^{\prime \prime}$ which contains $\gtrsim 90 \%$ of the source photons, using the encircled energy function given by Ghizzardi \& Molendi (2001). For the PN camera we used a rectangle (in RAW pixels) of $\sim 110^{\prime \prime} \times 110^{\prime \prime}$ as extraction region around the source, avoiding the chip boundary. In the case of PG $1411+442$ we also ignored a nearby weak source. Only single and double events (i.e. with pattern 0-4 for the PN camera and 0-12 for the MOS cameras) and with quality flag 0 were chosen. The backgrounds were determined with the same selection criteria from source free regions on the same chips.

\subsection{The light curves}

Figure 1 shows the co-added, background subtracted MOS + PN 0.2-10 keV light curve for PG $1411+442$. The time bin 


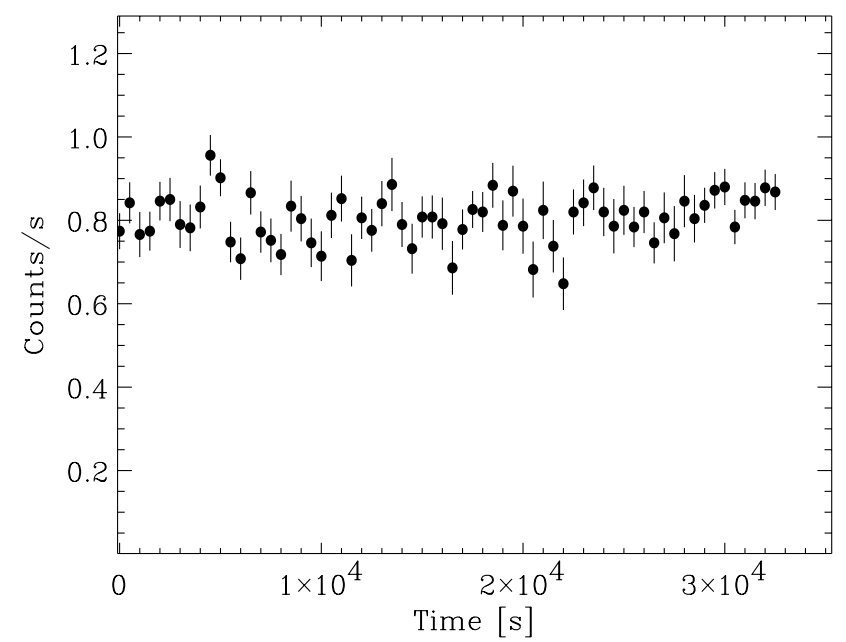

Fig. 2. Combined PN + MOS, background subtracted, 0.2-10 keV light curve of Mrk 304; the time binning is $500 \mathrm{~s}$, the time is counted from the start of the PN observation.

size was set to be $500 \mathrm{~s}$. As the PN camera was switched on about $9.5 \mathrm{ksec}$ later than the MOS cameras the first part of the light curve (open symbols) represents the summed count rates of the MOS only. The light curve is flat with no obvious source intrinsic variability but with some prominent spikes towards the end of the observation which are caused by large background flares. In the following spectral analysis we will ignore these periods by filtering them out via a GTI file and, in general, all data taken at times $>37 \mathrm{ksec}$ after the start of the observation.

Figure 2 shows the co-added, background subtracted MOS + PN 0.2-10 keV light curves for Mrk 304, using the same time bin size as above. During this observation the background was high and quite noisy but without strong flares. The PN camera was switched on about $2.85 \mathrm{ksec}$ later than the MOS cameras but as the MOS data suffer from high background at the beginning of the observation we start the light curve when all instruments are switched on. Again, the source flux appears to be at a constant level during the observation.

\section{Spectral analysis}

Our analysis is mainly based on the PN data, as they have a larger signal to noise (compared to MOS data) and a wider bandpass. Due to the small count rate of both sources, pile-up is not an issue for the EPIC-PN data. We binned the spectra to contain at least 40 counts per energy channel and screened the data according to different levels of background activity from the GTI files. However, this influenced the spectral fits only marginally; the fitted model parameters did not show any statistically significant changes.

We have used XSPEC v11.2.90 to perform the spectral fitting analysis. The errors on the best-fitting parameter values correspond to the $90 \%$ confidence limits for one interesting parameter (i.e. they were found using $\Delta \chi^{2}=\chi^{2}+2.706$ ). Furthermore, we have adopted the $90 \%$ probability limit in assessing whether a best fitting model is statistically acceptable, and the $95 \%$ confidence level in assessing (with the use of $F$-test) whether or not the addition of new features improves

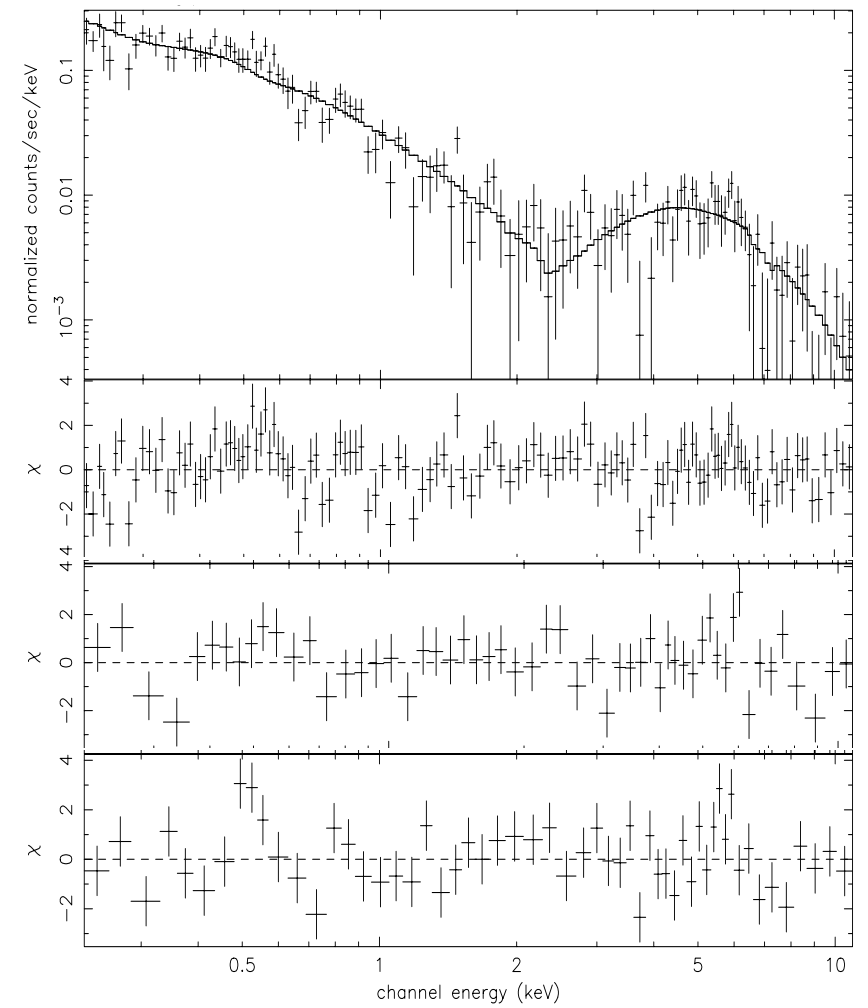

Fig. 3. Fit of two power laws with galactic absorption to the data of PG $1411+442$. The hard power law is additionally absorbed by source intrinsic material. The upper two panels show the PN fit and the $\Delta \chi^{2}$ per channel. The lower two panels give the $\Delta \chi^{2}$ per channel for the corresponding MOS 1 and MOS 2 fits, respectively.

the goodness of fit of a particular model. To compare different models we have used the "likelihood ratio" (Mushotzky 1982) and accepted values of the ratio larger than 100 as showing significant improvement in the model fits. Finally, all energies quoted refer to the emitter's rest-frame.

\section{1. $P G 1411+442$}

First, we fitted the PN spectrum with the best fitting model from a joint fit to the ROSAT - ASCA spectra (Brinkmann et al. 1999). This "Model 0" consists of a heavily absorbed power law (with the absorber being intrinsic to the source) and an extra power law like component at energies below $\sim 2 \mathrm{keV}$ to account for the soft excess emission from the source. Both components are further absorbed by galactic material with $N_{\mathrm{H}}$ fixed at $1.05 \times 10^{20} \mathrm{~cm}^{-2}$ (Lockman \& Savage 1995). Although the model formally does not provide an acceptable fit $\left(\chi_{\text {red }}^{2}=1.324\right.$ for 132 d.o.f.) it represents the global shape of the spectrum quite well. The best fitting model together with the residual plot are shown in Fig. 3 in the upper two panels. We then fitted the same model to the MOS data and present the residuals in third panel (MOS1) and in the bottom panel (MOS2). The fit yielded for the MOS $1 \mathrm{a} \chi_{\text {red }}^{2}=1.27$ for 51 d.o.f., for the MOS2 $\mathrm{a} \chi_{\text {red }}^{2}=1.7$ for 51 d.o.f., respectively. The fitted parameters are given in Table 1.

As Fig. 3 shows, the spectra are rather noisy. In particular, the count rates in the two MOS cameras are considerably 
Table 1. Results of spectral fits to the PG $1411+442$ data.

\begin{tabular}{|c|c|c|c|c|c|c|c|c|c|c|}
\hline Model & $\Gamma_{\text {soft }}$ & $\Gamma_{\text {hard }}$ & $\begin{array}{c}N_{\mathrm{H}} \\
\left(\times 10^{22} \mathrm{~cm}^{-2}\right)\end{array}$ & $\mathrm{CF}$ & $\begin{array}{l}E_{\text {line }} \\
(\mathrm{keV})\end{array}$ & $\begin{array}{c}E_{\text {edge }} \\
(\mathrm{keV})\end{array}$ & $\tau$ & $\xi$ & $\chi_{\text {red }}^{2}$ & d.o.f \\
\hline $0 / \mathrm{PN}$ & $2.94_{-0.07}^{+0.07}$ & $1.94_{-0.52}^{+0.52}$ & $12.8_{-3.8}^{+3.8}$ & - & - & - & - & - & 1.324 & 132 \\
\hline $0 / \mathrm{M} 1$ & $2.71_{-0.15}^{+0.23}$ & $0.66_{-0.41}^{+0.75}$ & $4.4_{-2.0}^{+7.4}$ & - & - & - & - & - & 1.273 & 51 \\
\hline $0 / \mathrm{M} 2$ & $2.87_{-0.16}^{+0.15}$ & $2.08_{-0.16}^{+0.61}$ & $20.8_{-10.3}^{+11.9}$ & - & - & - & - & - & 1.698 & 51 \\
\hline A & $2.85_{-0.14}^{+0.16}$ & $2.25_{-0.9}^{+0.1 .2}$ & $18.5_{-9.5}^{+8.0}$ & - & $\begin{array}{l}0.58_{-0.05}^{+0.03} \\
6.43_{-0.04}^{+0.07}\end{array}$ & - & - & - & 1.078 & 126 \\
\hline B-1 & - & $2.48_{-0.13}^{+0.21}$ & $25.6_{-12.8}^{+8.7}$ & $0.95_{-0.12}^{+0.02}$ & $6.43 \pm 0.05$ & $\begin{array}{l}0.69 \pm 0.03 \\
1.04 \pm 0.05\end{array}$ & $\begin{array}{l}0.65_{-0.23}^{+0.26} \\
1.25_{-037}^{+0.57}\end{array}$ & - & 1.086 & 126 \\
\hline B-2 & - & $2.63_{-0.56}^{+0.56}$ & $15.6_{-2.1}^{+2.1}$ & $0.96_{-1.0}^{+1.0}$ & 0.58 & - & $-{ }^{-0.37}$ & - & 1.162 & 129 \\
\hline $\mathrm{C}^{1}$ & - & $\begin{array}{r}1.87_{-0.30}^{+0.23} \\
\end{array}$ & $53.2_{-25.4}^{+11.3}$ & - & 6.43 & - & - & $1000_{-725}^{+2100}$ & 0.936 & 58 \\
\hline
\end{tabular}

${ }^{1}$ Model $\mathrm{C}$ results refer to the best model fit of the $2-11 \mathrm{keV}$ spectrum only. The line energy was fixed at the best fit value of Model A.

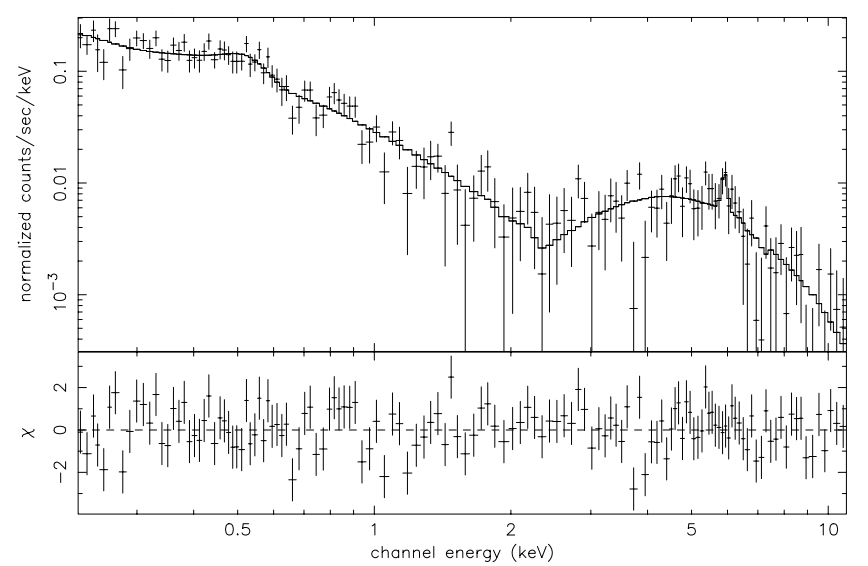

Fig. 4. The same fit of two power laws as in Fig. 3 with the addition of two Gaussian lines.

smaller $(0.037 \mathrm{cts} / \mathrm{s}$ for the MOS1, $0.033 \mathrm{cts} / \mathrm{s}$ for the MOS2, respectively) than for the PN $(0.127 \mathrm{cts} / \mathrm{s})$, resulting in substantially larger uncertainties for the fitted parameters. For example, the MOS1 best fit parameters seem to be quite different when compared to the PN and MOS2 best fit parameters listed in Table 1. However, due to the large errors, these differences are statistically not significant. Most importantly though, inspection of the residuals reveals the presence of broadly similar structures in all three cameras, in particular, the presence of two emission features at $\sim 0.55 \mathrm{keV}$ and $\sim 6 \mathrm{keV}$.

Therefore, we added a Gaussian line with energy $\sim 0.6 \mathrm{keV}$ to Model 0 and repeated the fitting using the PN data only. The addition of this line improved significantly the quality of the fit $\left(\Delta \chi^{2}=28\right.$ for 3 d.o.f.). We then added a second Gaussian line with energy $\sim 6.4 \mathrm{keV}$. The goodness of fit further improved, $\left(\Delta \chi^{2}=7.7\right.$ for 3 d.o.f.) which implies an improvement at the $93 \%$ level according to an $F$-test. This model with two Gaussian lines (Model A) provides now a good fit to the spec$\operatorname{trum}\left(\chi_{\text {red }}^{2}=1.078 / 126\right.$ d.o.f. $)$ as Fig. 4 clearly shows. The best fitting model parameters are given in Table 1 . The slope of the heavily absorbed power law is not well determined due to the low signal at higher energies. The width of the two Gaussian lines is smaller than the intrinsic resolution of the PN camera at the respective energies $\left(\sigma_{\text {soft }} \sim 35 \mathrm{eV}, \sigma_{\text {hard }} \sim 10 \mathrm{eV}\right)$; their equivalent widths are $90_{-36}^{+32}$ and $250_{-230}^{+1200} \mathrm{eV}$, for the soft and hard band line, respectively.

Although the PN camera has a significantly higher count rate than the MOS1 and MOS2, it is possible that a joint model fit to all three spectra could reduce the uncertainties of the best fit parameters. Therefore, we performed a joint fit of Model A to the combined PN, MOS1 and MOS2 spectra. The resulting best fit parameters are very similar to the values listed in Table 1. However, the goodness of the fit $\left(\chi_{\text {red }}^{2}=1.33\right.$ for 238 d.o.f.) is significantly worse when compared to the best fit of the same model to the PN data only. The situation was not improved when we performed a joint fit of Model A to the PN plus MOS2 spectra only. The quality of the model fit $\left(\chi_{\text {red }}^{2}=1.31\right.$ for 184 d.o.f. $)$ is, again, worse than that of the same model fit to the PN spectrum. The residual plots do not reveal any systematic, well defined features that could be responsible for the low quality of the model fit to the PN, MOS1 and/or MOS2 data. They thus indicate perhaps only cross-calibration uncertainties and the different spectral responses of the instruments. Naturally, the errors of the individual model parameters decrease when we add the PN and MOS spectra in the model fit. But as for the combined data the quality of the model fits worsen significantly without obvious "residual" features, we chose to rely for the following fits on the PN data only.

The detection of an emission line in the soft band spectrum of PG $1411+442$ is indicative of the presence of hot, diffuse gas in the central region of the source. For that reason, we tried the meka thermal emission model to fit the soft band spectrum of the source (keeping the parameters of the intrinsic absorption, hard power law and iron emission line fixed at the best fit values of Model A). The model fails entirely to provide an acceptable fit to the spectrum. Therefore, we tried a combination of a power law plus a meka model. In this case, we do get an acceptable fit $\left(\chi_{\text {red }}^{2}=1.073\right.$, for 129 d.o.f.). With solar abundances the best fit gas temperature is $k T=0.14_{-020}^{+0.15} \mathrm{keV}$, and the best fit soft power law slope is $\Gamma_{\text {soft }}=2.64_{-0.22}^{+0.19}$. However, we get a good fit to the spectrum only when we let the redshift of the emitting gas as a free parameter. The best fit value we find, $\left(0.06_{-0.044}^{+0.040}\right)$, suggests a possible outflow at a speed of $\sim 8000 \mathrm{~km} \mathrm{~s}^{-1}$. 


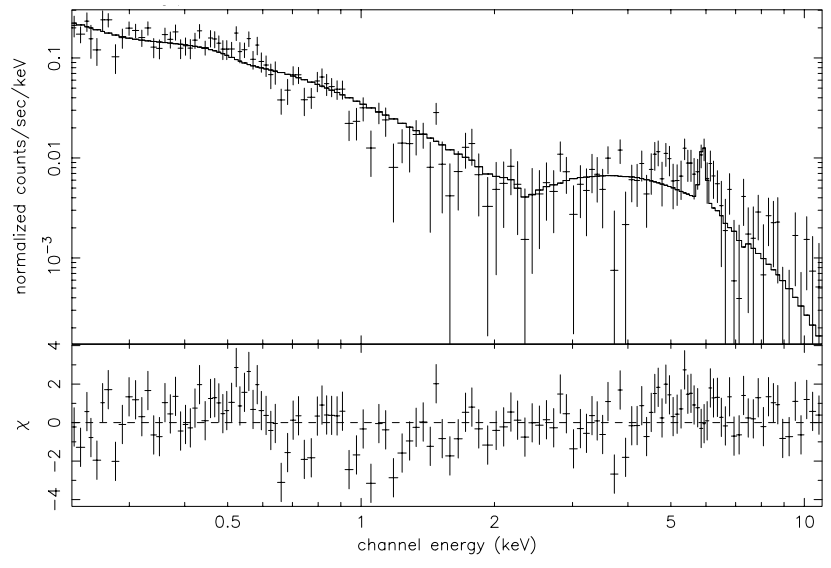

Fig. 5. Fit of a partially covering absorber plus iron line to PG $1411+442$. The lower panel gives the $\Delta \chi^{2}$ per channel.

In order to investigate whether a partial covering model can also describe the observed spectrum, we fitted the data with a power law plus a partially covering neutral absorber (model "zpcfabs" in XSPEC). We included a Gaussian line at $\sim 6.4 \mathrm{keV}$ to take account of the Iron $\mathrm{K} \alpha$ emission. The best fit model together with a plot of the residuals is shown in Fig. 5. The model does not provide a good fit to the data $\left(\chi_{\text {red }}^{2}=1.476\right.$ for 131 d.o.f.). Inspection of the residuals shows again the presence of an emission-like feature at $\sim 0.5 \mathrm{keV}$, and various absorption features at $\sim 0.75 \mathrm{keV}$ and $\sim 1 \mathrm{keV}$.

The model can fit well the spectrum if we consider the addition of two absorption edges (Model B-1) or of one emission line with its rest frame energy kept fixed at $0.58 \mathrm{keV}$ (i.e. the best fit value of the soft band line from Model A), which we call Model B-2. In the first case, the resulting $\chi_{\text {red }}^{2}$ value is 1.086 for 126 d.o.f. The absorber covers $95 \%$ of the central source, the column density is quite large $\left(N_{\mathrm{H}} \sim 2.6 \times 10^{23} \mathrm{~cm}^{-2}\right)$ and the best fit spectral slope of the underlying power law is steep $(\Gamma \sim 2.5)$.

In the the case of Model B-2, the fit is also acceptable with $\chi_{\text {red }}^{2}=1.16$ for 129 d.o.f. The best fitting values of the continuum parameters (e.g. covering fraction, power spectral slope, $N_{\mathrm{H}}$ ) are consistent with the values of Model B-1. The emission line at $\sim 0.6 \mathrm{keV}$ was found again to be narrow $(\sigma \sim 40 \mathrm{eV})$. However, comparison of the goodness of fit of models A, B-1 and B-2 with the use of the likelihood ratio implies that both Models A and B-1 fit the data significantly better when compared to Model B-2.

Since the detection of absorption edges (Model B-1) indicates the presence of ionized (i.e. "warm" material) distributed along the line of sight to the source, we also fitted the data with the ionized absorber model absori model of Zdziarski et al. (1995) (which is based on an earlier model of Done et al. 1992). The model fit was done only to the $2-11 \mathrm{keV}$ data. Over this energy range the model provides a good fit to the data (see the hard band of Fig. 6) with $\chi_{\text {red }}^{2}=0.936 / 58$ d.o.f., with the $\mathrm{Fe}$ abundance and temperature fixed at the solar value and at $T=3 \times 10^{4} \mathrm{~K}$, respectively. The resulting best fit model parameters given in Table 1 indicate a flat power law and high column density.

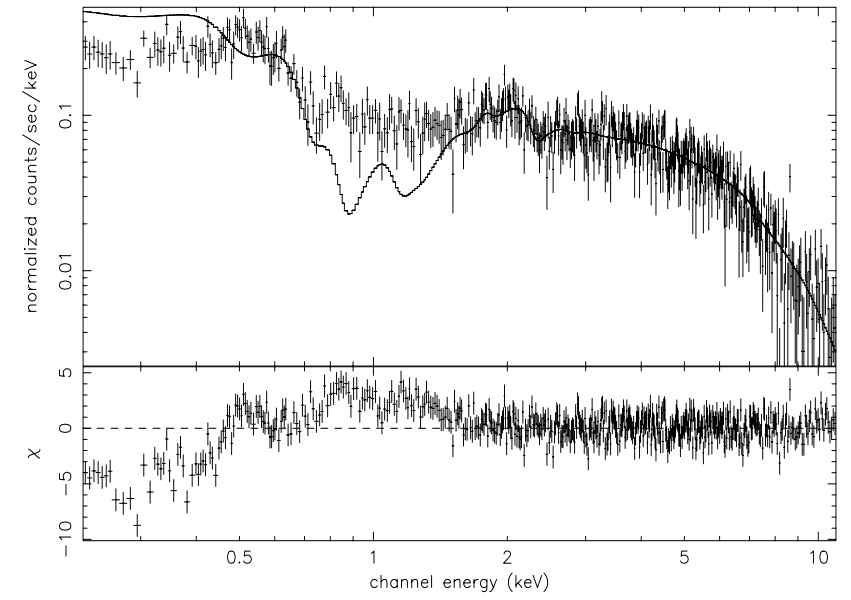

Fig. 6. Fit of the absori model with galactic absorption to the PN data of PG $1411+442$. The model was fitted to the $2-11 \mathrm{keV}$ band only; data and model extrapolated to lower energies. The lower panel gives the $\Delta \chi^{2}$ per channel.

However, when we consider the soft band $(0.2-2 \mathrm{keV})$ data as well, the model does not provide a good fit to the broad band spectrum. The extrapolation of the model to soft energies reveals large deviations between model and data (Fig. 6), which we were not able to fit by any combination of standard XSPEC models. If the absorbing material is indeed ionized, this result implies that it might have a complex ionization structure. There could be multiple layers of matter with different ionization states, perhaps not even completely covering the whole source.

\subsection{Mrk 304}

As with PG 1411+442, we first tried for Mrk 304 a model consisting of two power law components, one absorbed by neutral material at the source's redshift plus galactic absorption $\left(N_{\mathrm{H}}=5.23 \times 10^{20} \mathrm{~cm}^{-2}\right.$, Elvis et al. 1989), and the other absorbed by the galactic neutral material only (Model 0 ). The model does not provide a good fit to the PN data $\left(\chi_{\text {red }}^{2}=1.29\right.$ for 506 d.o.f.). The fit and the residuals are shown in the upper two panels of Fig. 7, the numerical values in Table 2. Similar parameters were obtained from fits of the same model to the $\operatorname{MOS} 1$ data $\left(\chi_{\text {red }}^{2}=1.33\right.$ for 133 d.o.f.) and the MOS2 data $\left(\chi_{\text {red }}^{2}=1.27\right.$ for 133 d.o.f. $)$. The residuals are given in the two lower panels of Fig. 7. These residuals of all three instruments indicate, as in the case of PG $1411+442$, the presence of a strong emission feature at $\sim 0.55 \mathrm{keV}$ and of an absorption edge-like feature at around $\sim 0.7 \mathrm{keV}$. As with PG $1411+442$ we use only the PN data when we investigate more complicated models.

Thus we added a Gaussian component in Model 0, to account for the emission feature. The new model provides a formally acceptable fit to the data $\left(\chi_{\text {red }}^{2}=1.08\right.$ for 504 d.o.f.). The further addition of an edge provides a statistically significant improvement to the goodness of fit. We therefore conclude that Model 0 fits well the spectrum only if we add a Gaussian line and an edge (Model A). The best fitting model $\left(\chi_{\text {red }}^{2}=1.062\right.$ 
Table 2. Results from spectral fits to the Mrk 304 data.

\begin{tabular}{llccccccccc}
\hline \hline Model & $\Gamma_{\text {soft }}$ & $\Gamma_{\text {hard }}$ & $\begin{array}{c}N_{\mathrm{H}} \\
\left(\times 10^{22} \mathrm{~cm}^{-2}\right)\end{array}$ & $\mathrm{CF}$ & $\begin{array}{c}E_{\text {line }} \\
(\mathrm{keV})\end{array}$ & $\begin{array}{c}E_{\text {edge }} \\
(\mathrm{keV})\end{array}$ & $\tau$ & $\xi$ & $\chi_{\text {red }}^{2}$ d.o.f. \\
\hline 0 / PN & $2.86_{-0.06}^{+0.06}$ & $1.44_{-0.06}^{+0.06}$ & $2.7_{-0.17}^{+0.17}$ & - & - & - & - & - & 1.296 & 506 \\
$0 / \mathrm{M} 1$ & $2.89_{-0.20}^{+0.18}$ & $1.33_{-0.14}^{+0.16}$ & $2.7_{-0.4}^{+0.6}$ & - & - & - & - & - & 1.326 & 133 \\
$0 / \mathrm{M} 2$ & $3.02_{-0.20}^{+0.19}$ & $1.22_{-0.15}^{+0.14}$ & $2.3_{-0.5}^{+0.4}$ & - & - & - & - & - & 1.263 & 133 \\
$\mathrm{~A}$ & $2.42_{-0.15}^{+0.15}$ & $1.52_{-0.12}^{+0.11}$ & $3.7_{-0.5}^{+0.4}$ & - & $0.59_{-0.02}^{+0.02}$ & $0.72_{-0.18}^{+0.04}$ & $0.50_{-0.26}^{+0.25}$ & - & 1.062 & 502 \\
$\mathrm{~B}$ & - & $1.54_{-0.10}^{+0.14}$ & $4.0_{-1.3}^{+3.9}$ & $0.72_{-0.07}^{+0.10}$ & $0.61_{-0.18}^{+0.09}$ & $0.72_{-0.02}^{+0.01}$ & $>5.8$ & - & 1.070 & 502 \\
$\mathrm{C}^{1}$ & - & $1.60_{-0.17}^{+0.17}$ & $6.6_{-2.7}^{+5.2}$ & - & - & - & - & $93_{-79}^{+264}$ & 1.089 & 330 \\
\hline
\end{tabular}

${ }^{1}$ Model C fitting results refer to the best model fitting of the $2-11 \mathrm{keV}$ spectrum only.

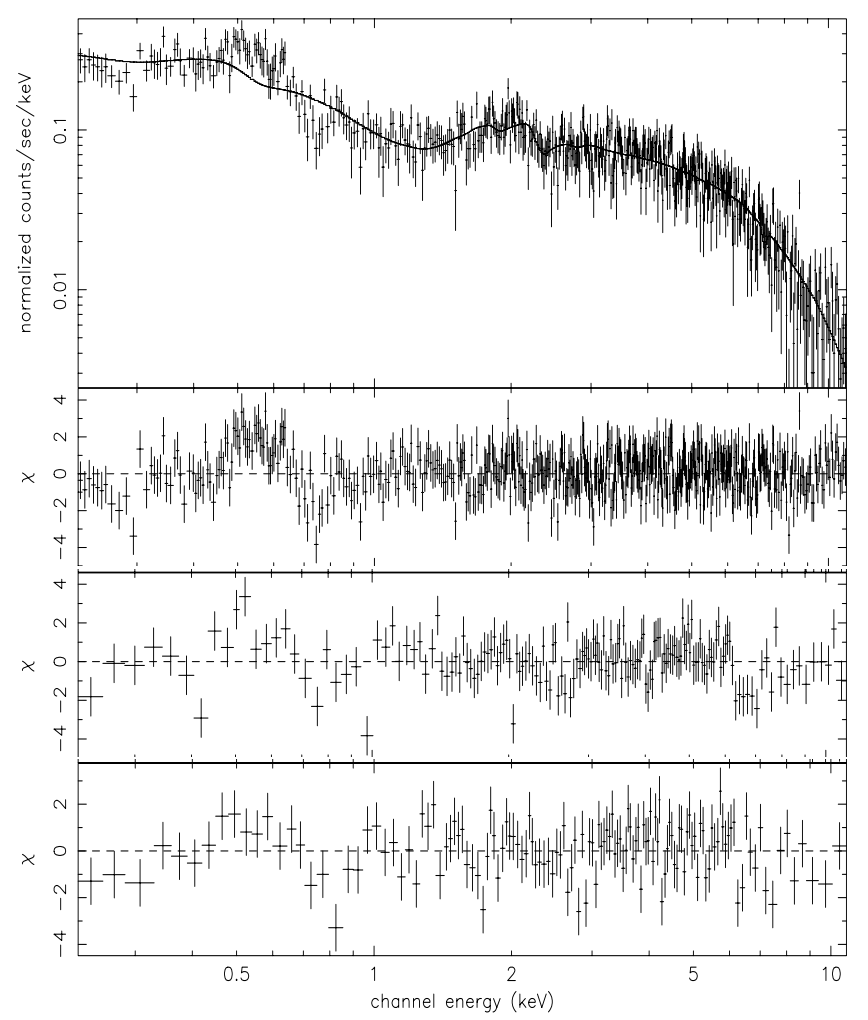

Fig. 7. Fit of two power laws with galactic absorption to the data for Mrk 304. The hard power law is additionally absorbed by source intrinsic material. The upper two panels give the PN fit and the $\Delta \chi^{2}$ per channel. The lower two panels give the residuals in $\Delta \chi^{2}$ per channel for the corresponding MOS1 and MOS2 fits.

for 502 d.o.f.) is given in Fig. 8, and the respective parameter values are listed in Table 2. The equivalent width of the soft line is $E W \sim 120 \mathrm{eV}$. There is no sign of significant emission around $\sim 6.4 \mathrm{keV}$ in Mrk 304. In fact, adding a line to the model decreases the quality of the fit. We obtain an upper limit for the $E W$ of a narrow iron line of $\sim 30 \mathrm{eV}$ with very large errors. Compared to PG $1411+442$ both, the soft-band and the hard-band, power laws are considerably harder by a factor of $\Delta \Gamma \sim 0.5-0.7$.

Since the presence of emission features in the soft band spectrum of Mrk 304 is suggestive for the presence of hot gas in the source, we fitted the spectrum with a combination of a power law plus a meka model, keeping the intrinsic absorption

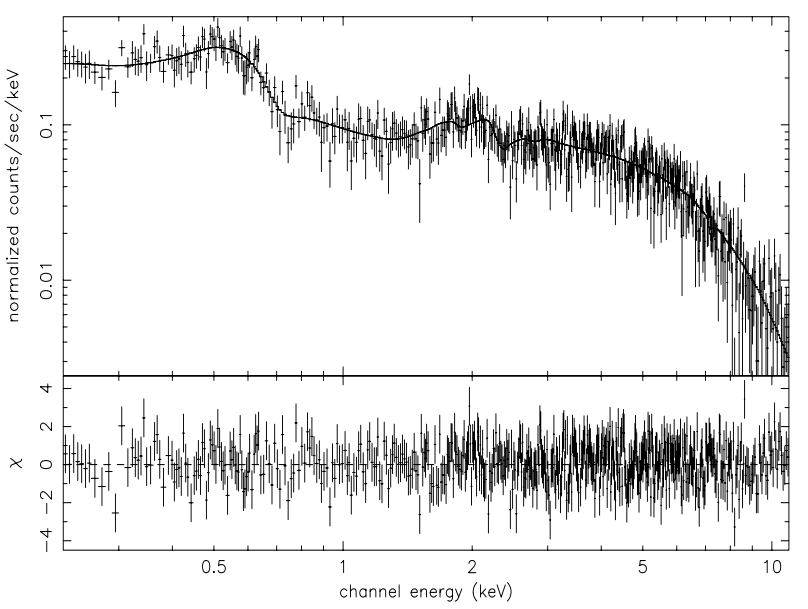

Fig. 8. Fit of two power laws as in Fig. 7 plus a soft Gaussian line and an edge. The lower panel gives the $\Delta \chi^{2}$ per channel.

and hard band power law model parameters fixed at their best Model A fitting values. The new model does not describe the spectrum well $\left(\chi_{\text {red }}^{2}=1.1\right.$ for 504 d.o.f.). The main reason for this is the presence of an absorption feature around $\sim 0.7 \mathrm{keV}$. We therefore added an absorption edge and repeated the model fit, keeping the edge's redshift "tied" to the fitted redshift of the meka emission spectrum. This model provides an acceptable fit to the spectrum $\left(\chi_{\text {red }}^{2}=1.054\right.$ for 502 d.o.f.). The best fit temperature of the hot gas is $k T=0.30_{-0.02}^{+0.06}$. The best fit edge energy is $0.65_{-0.01}^{+0.07}$, and the resulting soft power law slope is $\Gamma_{\text {soft }}=2.10_{-0.21}^{+0.15}$. The edge's optical depth is not well constrained (we could find a lower limit only, i.e. $\tau>7.1$ ). The best fit redshift value $(\sim 0.03 \pm 0.03)$, although not well constrained, indicates that the gas moves at a speed of $\sim 3500 \mathrm{~km} \mathrm{~s}^{-1}$.

We also investigated whether a neutral partially covering absorber can provide an acceptable fit to the spectrum. A model which consists of a power law, partially absorbed by material in the source (taking into account the effects of the galactic absorption as well) does not fit the data well $\left(\chi_{\text {red }}^{2}=1.7\right.$ for 508 d.o.f.). The residuals show again the presence of both an emission and an absorption feature in the soft energy band. We therefore repeated the fit with the addition of a Gaussian line and an absorption edge (Model B). The model fits the data similarly well $\left(\chi_{\text {red }}^{2}=1.07\right.$ for 502 d.o.f. $)$ as Model A. The best fitting line centroid energy, and edge energy are consistent with the respective values for best fit results of Model A. 


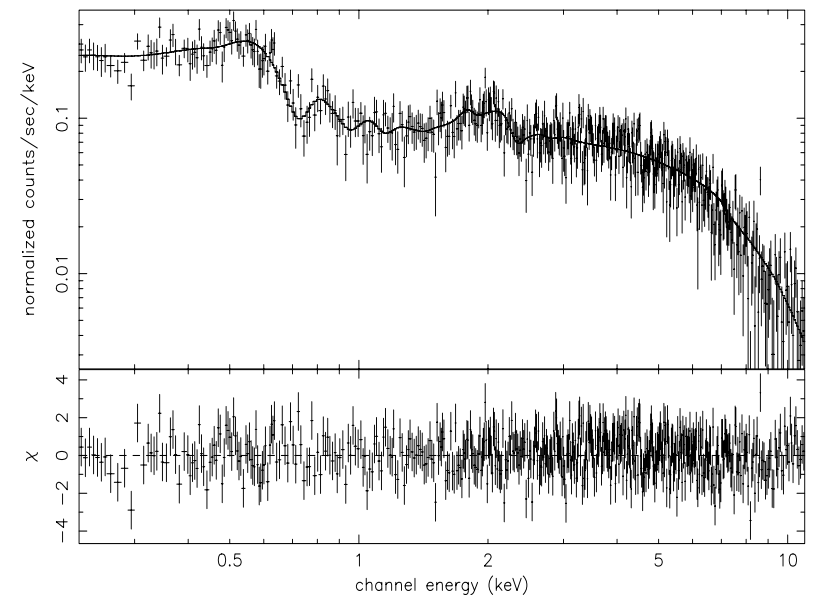

Fig. 9. Fit of an absori plus three Gaussian lines model to the $0.2-11 \mathrm{keV}$ energy band of Mrk 304. The lower panel gives the $\Delta \chi^{2}$ per channel.

The edge's optical depth though appears to be significantly larger (although not well constrained, we find that $\tau>5.4$ ) than in the case of PG $1411+442$, and the line width is also significantly larger $\left(\sigma=0.36_{-0.11}^{+0.29}\right)$.

Finally, since we detect evidence for absorption from ionized oxygen, we also tried to fit the data with the ionized absorber model absori. As in the case of PG 1411+442, we fitted the model to the $2-11 \mathrm{keV}$ spectrum only. The model describes this part of the spectrum well $\left(\chi_{\text {red }}^{2}=1.09\right.$ for 330 d.o.f.), with $N_{\mathrm{H}} \sim 6.6 \times 10^{22} \mathrm{~cm}^{-2}$ and $\xi \sim 100$. However, when the model is extrapolated over the full energy band it fails to describe the soft band data, mainly due to the presence of three localized regions of strong residuals. If we add at these energies Gaussian lines and leave all relevant parameters free we obtain an acceptable fit with $\chi_{\text {red }}^{2}=1.071$ for 497 d.o.f.

Figure 9 shows the fit and the remaining residuals. The line centroids are at energies of $E_{1}=0.575 \pm 0.011 \mathrm{keV}$, $E_{2}=0.899 \pm 0.009 \mathrm{keV}$, and $E_{3}=1.312 \pm 0.037 \mathrm{keV}$; the lines are all narrow with widths similar to the corresponding errors. The best fit power law slope is $\Gamma=1.305 \pm 0.041$, the ionization parameter $\xi=103.1 \pm 28.2$, and the relative iron abundance $2.53 \pm 0.59$. This implies that, if there is indeed absorption from ionized material, the physical state of this material (i.e. the ionization parameter, the covering fraction, the density) is not constant; it probably varies with the distance from the central source.

\section{Discussion}

The results from the spectral analyses of the quasars PG 1411+442 and Mrk 304 strongly support the view that the $\mathrm{X}$-ray weakness of the objects is caused by strong intrinsic absorption of the primary X-ray emission.

The X-ray spectra of both objects can be fitted well by a model which consists of a hard power law, intrinsically absorbed by matter with large column densities (around $N_{\mathrm{H}} \sim$ $2 \times 10^{23} \mathrm{~cm}^{-2}$ for PG $1411+442$ and $N_{\mathrm{H}} \sim 4 \times 10^{22} \mathrm{~cm}^{-2}$ in the case of Mrk 304), and a steep soft X-ray component. This component cannot be fitted by any of the frequently used thermal models (i.e. bremsstrahlung, black-body, ionized disc, or Meka models). However, soft X-ray components like the ones observed here can be produced by matter which lies out of the line of sight and scatters the intrinsic continuum radiation back into the line of sight. Simple power-law like continuum emission is appropriate in the case of pure electron scattering in a highly ionized continuum. In this case, the slope of the soft X-ray component should be similar to the slope of the primary continuum. Since this is not the case here (especially for Mrk 304) the ionization state of the scattering gas has to be taken into account in a realistic investigation (by including e.g. the effects of absorption and re-emission of recombination continua) of this possibility. However, the present spectra have insufficient photon statistics for a reliable detailed modeling.

In any case, the presence of "warm" (i.e. ionized) material in the central regions of the two objects is revealed by the presence of distinct emission and absorption features in their soft X-ray spectra. In both objects we find an emission feature which is narrow and its centroid energy has identical values of $\sim 590 \mathrm{eV}$. This energy is slightly in excess of the energies expected from the O VII triplet, but mostly compatible with it inside the fitting errors. The fitted line position further depends on the underlying continuum and the limited photon statistics does not allow to distinguish whether the shift of the line energy is a result of the data fitting of whether we see the blueshifted emission from out-flowing matter from the sources. The O VII lines are among the strongest lines expected in the soft X-ray band, and their predicted equivalent width $(\sim 50 \mathrm{eV}$; Netzer 1996) is consistent with the equivalent width of the lines we observe in both objects.

A combined power law plus meka model fits well the soft band spectra of both sources. This result supports the idea that, in both sources, the soft band emission line is due to the presence of hot gas (with $k T \sim 0.15-0.3 \mathrm{keV}$ ), which may be moving out of the sources at speeds of $\sim 3000-10000 \mathrm{~km} \mathrm{~s}^{-1}$. The addition of this thermal component decreases the differences between the best fit values of $\Gamma_{\text {soft }}$ and $\Gamma_{\text {hard }}$, although it does not eliminate them completely (especially in the case of Mrk 304).

Perhaps the dominant effect of ionized gas on the soft X-ray spectrum is absorption by this material along the line of sight. Both sources show strong evidence for absorption structures around $\sim 0.7 \mathrm{keV}$ and $\gtrsim 1 \mathrm{keV}$ (in the case of PG $1411+442$ ). The presence of highly ionized, absorbing gas which reveals absorption edges attributable to oxygen is confirmed for numerous Seyfert 1 galaxies (see, for example, George et al. 1998). The edge energies of $0.69 \mathrm{keV}$ fitted for PG $1411+442$ and $0.72 \mathrm{keV}$ for Mrk 304 are close to, but smaller than, the OVII absorption edge energy of $739 \mathrm{eV}$. A deficit in the counts at $\sim 1 \mathrm{keV}$ found for several ASCA sources was interpreted as the result of absorption by a second component of ionized gas along the line of sight (Otani et al. 1996; Kriss et al. 1996). For PG $1411+442$ we find an edge energy of $1.04 \mathrm{keV}$, in between the OVIII $(871 \mathrm{eV})$ and NeIX (1196 eV) edge energies, and consistent with the position of the $\mathrm{Ne} \mathrm{X} \operatorname{Ly} \alpha$ absorption line at $1.023 \mathrm{keV}$.

Although the inclusion of these edges improve the quality of the fits considerably the physical interpretation of their exact nature (e.g. the identification of the atoms responsible for the 
absorption) remains uncertain. The residuals of the above fits indicate that data with considerably better signal to noise are required for more accurate parameter estimations. Nevertheless, the main result of this work, i.e. the unambiguous detection of both emission and absorption features in the soft X-ray spectra of the two objects, strongly supports the idea that at least some of the absorbing material must be ionized. Interestingly, the warm absorber model absori provides an excellent fit to the data, but only at energies $\gtrsim 1.5 \mathrm{keV}$. At lower energies it fails completely and the remaining residuals cannot be modeled with any reasonable combination of standard XSPEC models (except from the addition of three emission lines in the case of Mrk 304).

We conclude that, although the evidence for the presence of ionized material is strong, the absorber is complex (perhaps the ionization state is not uniform, or perhaps the ionized absorber is only partially covering the source) and neither the quality of the present data nor the use of simple models can account for all the spectral features that we observe at low energies.

Comparing the results from the best fit models to the PG 1411+442 and the Mrk 304 spectra we note some similarities but important differences as well. We discuss below in some detail the spectral analysis results for the two sources individually.

\section{1. $P G 1411+442$}

Extrapolating the hard absorbed power law to soft X-ray energies yields an unabsorbed value for the X-ray loudness of PG $1411+442$ of $\alpha_{\text {ox }}=1.62$, which is typical for radio-quiet quasars (Yuan et al. 1998a). Thus, strong intrinsic absorption can quite well account for the X-ray weakness of this source.

The total luminosity in the XMM band amounts to $L_{0.2-10 \mathrm{keV}} \sim 1.5 \times 10^{44} \mathrm{erg} \mathrm{s}^{-1}$, of which a substantial fraction is emitted in the soft band, $L_{0.2-2 \mathrm{keV}} \sim 1.1 \times 10^{44} \mathrm{erg} \mathrm{s}^{-1}$. Estimating the count rates using PIMMS we find that the source intensity has not changed compared to previous ROSAT (Rachen et al. 1996) and ASCA (Brinkmann et al. 1999) observations.

While strong intrinsic absorption is obviously responsible for the X-ray weakness of PG 1411+442 different fitted models can, to a certain degree, account for this absorption, resulting in different physical scenarios.

The model of a simple power law, intrinsically heavily absorbed by cold material, has the attractiveness that the power law slope of $\Gamma \sim 2$ is a value, generally found for radioquiet quasars (Brinkmann et al. 1997; Reeves \& Turner 2000). However, an additional second, much steeper soft power law component, which accounts for a few percent of the intrinsic flux at $1 \mathrm{keV}$, is required to explain the soft excess emission which further exhibits some emission and absorption structures. As discussed above and by Brinkmann et al. (1999), this component can be attributed to scattering. In fact, reflection models (cf. Ross \& Fabian 1993; Netzer et al. 1998; Ballantyne et al. 2003) are also able to reproduce this kind of spectra. However, in this case, the geometry of the absorbing material has to be rather special, as the neutral absorbing material must

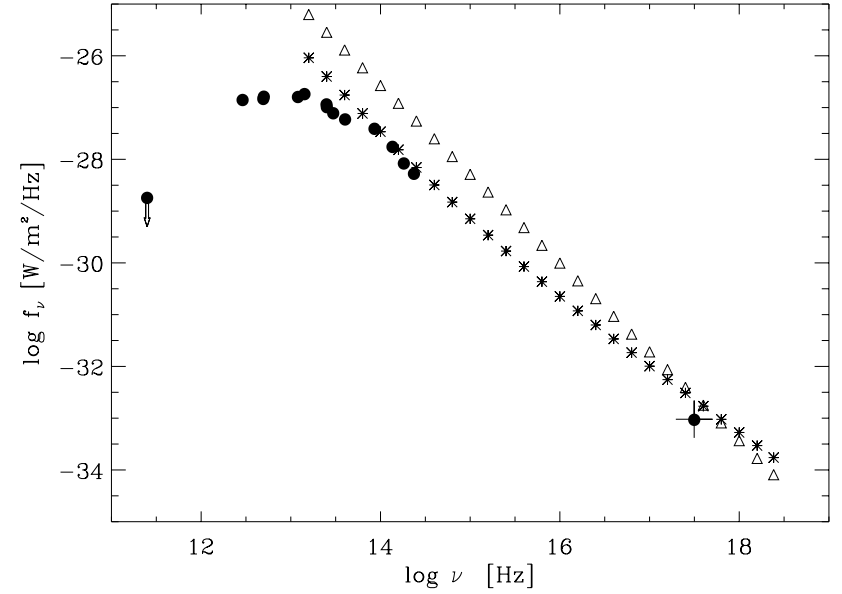

Fig. 10. Infrared-optical SED of PG $1411+442$, based on previous flux measurements (full dots); the ROSAT point is indicated by a cross. Triangles are the extrapolation of the partial covering model, stars of the two power law models towards the optical band (without absorption).

have the right size to cover the central X-ray source, but not the emission from the reflecting disc.

A partial covering model describes the data equally well, requiring again some emission and absorption features at low energies. Only one emission region, covered to $\sim 95 \%$ by cold matter can account for the general spectral behavior of the source. However, the drawback of this otherwise attractive model is the inference that the intrinsic power law is very steep, $\Gamma \gtrsim 2.6$, which would be highly unusual for a radio-quiet quasar and indicate some other peculiarities in this source.

Figure 10 shows the infrared-optical SED of PG $1411+442$ based on previously measured fluxes (full dots) and the extrapolation into the optical band of the above two models (two power laws: stars; partial covering model: triangles). The ROSAT data point is additionally marked with a cross. Interestingly, the extrapolation of the two power law model, not considering absorption, perfectly matches the optical data. The partial covering model over predicts the optical flux by nearly a factor of 10 , which favors again the scattering model for the X-ray data of PG $1411+442$.

The best fitting line energy in the hard band suggests iron emission by fluorescence in near neutral material. The equivalent width of the line is slightly larger than the average value found by Nandra \& Pounds (1994) from a fit of narrow Gaussians to GINGA spectra of Seyfert 1 galaxies. The slightly larger equivalent width might be caused by the obscuration of the primary continuum by the neutral absorbing material with large column density. Both the spectral slope of $\Gamma \sim 2$ and the characteristics of the iron line suggest that at least the $2-10 \mathrm{keV} \mathrm{X}$-ray spectrum of the source is similar to the average X-ray spectrum of Type I AGN.

The fact that strong absorption by cold and/or warm material is the main cause for the $\mathrm{X}$-ray weakness of PG $1411+442$ is in agreement with the results for the spectral features at low energies in this source. These features also indicate that the absorber is complex, and that the spectral characteristics result from specific geometrical conditions of the 
accretion flow and from a particular viewing condition of the observer with respect to the quasar. PG $1411+442$ was classified as a BAL quasar although it possibly does not represent a "bona fide" object of its class. A popular model for the BAL phenomenon predicts a line of sight close to the equatorial plane, so that the primary X-ray flux is attenuated by a strong disc wind (Murray et al. 1995). Interestingly the inclination of the galaxy was determined to be $\sim 57$ degrees. Thus, either the accretion disc is not aligned with the plane of the galaxy or we are observing a geometrical configuration as proposed by Elvis (2000) where the wind, rising vertically from the disk gets bent radially under the influence of the radiation pressure from the quasar continuum.

Finally, Surace et al. (2001) note from optical and IR observations that the morphology of PG $1411+442$ is consistent with a minor merger and they find a tidal loop, which extends to the north, wraps around to the east, and extends south. In the nearinfrared, as well as in the model-subtracted optical images, a linear structure is seen extending to the southwest from the nucleus, which may be a jet. These findings indicate that there is large scale gas flow in the system which could be responsible for the strong absorption.

\section{2. $M r k 304$}

For Mrk 304 the extrapolation of the hard absorbed power law to soft X-ray energies yields an unabsorbed value for the X-ray loudness of $\alpha_{\mathrm{ox}}=1.503$, again a value typical for radio-quiet quasars (Yuan et al. 1998a). Therefore intrinsic absorption can account for the X-ray weakness of this source but it is possible that temporal variability plays an additional role as well. The ROSAT PSPC count rate was $\sim 0.014 \mathrm{cts} / \mathrm{s}$ (Rachen et al. 1996) while the count rate predicted from the above models by PIMMS would yield $\sim 0.074 \mathrm{cts} / \mathrm{s}$. The predictions for the IPC are $0.0365 \mathrm{cts} / \mathrm{s}$, somewhere in between the extreme values of $0.019 \mathrm{cts} / \mathrm{s}$ and $0.067 \mathrm{cts} / \mathrm{s}$ quoted for different Einstein pointings (Della Ceca et al. 1990). These values suggest large amplitude variability (of at least a factor of $\sim 5$ ) over a time scale of years.

The total luminosity in the XMM band amounts to $L_{0.2-10 \mathrm{keV}} \sim 1.14 \times 10^{44} \mathrm{erg} \mathrm{s}^{-1}$, most of which is emitted in the hard band, $L_{2-10 \mathrm{keV}} \sim 7 \times 10^{43} \mathrm{erg} \mathrm{s}^{-1}$.

The spectral energy distribution in the X-ray band is quite unusual for a radio-quiet quasar. In all fitted models the hard power law is extremely flat (typically around $\Gamma \sim 1.5$ ) and we don't find any indication for an iron line. The column density of the absorbing matter is only a few $\times 10^{22} \mathrm{~cm}^{-2}$ and the extrapolation of the X-ray model fits into the optical band in Fig. 11 does not favor any of the two models. The infrared-optical SED appears very flat and the morphology of the galaxy in the optical is very smooth and round and its profile does not fit an exponential or $r^{1 / 4}-$ law (Hutchings \& Neff 1992). It is a very blue, very compact object and shows considerable night to night variations in the optical (Jang \& Miller 1995).

The best fitting $\Gamma$ values of both the hard and soft power law components are significantly flatter than the respective values of PG 1411+442. Consequently, although our results show the

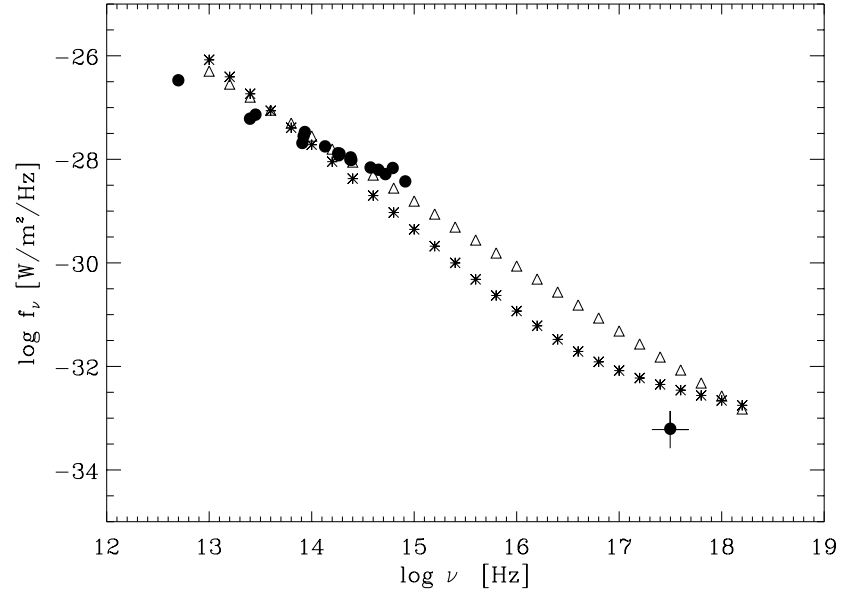

Fig. 11. Previous flux measurements from Mrk 304 (full dots); the ROSAT point is further indicated by a cross. Triangles are the extrapolation of the partial covering model, stars of the two power law models towards the optical band (without absorption).

presence of substantial, intrinsic to the object, absorbing material in the line of sight, at the same time, the spectral properties of the source (flat power law, lack of iron $\mathrm{K} \alpha$ emission) support the view that Mrk 304 could be a member of a class of quasars with SED intrinsically different than the SED of "normal quasars" (Risaliti et al. 2003). If numerous, such objects with intrinsically flat spectra and intrinsic absorption could perhaps contribute significantly to the X-ray background radiation.

\section{Summary}

We have presented the results from the analysis of XMM-Newton observations of two X-ray weak quasars, namely PG $1411+442$ and Mrk 304. They are among the highest signal-to-noise X-ray observations of this type of objects so far. We find conclusive evidence for the presence of absorbing material in the rest frame of the sources. A hard power law component which is absorbed by a uniform neutral screen with a column density of $N_{\mathrm{H}} \sim 2 \times 10^{23} \mathrm{~cm}^{-2}$ and $N_{\mathrm{H}} \sim 4 \times 10^{22} \mathrm{~cm}^{-2}$ in the case of PG $1411+442$ and Mrk 304 respectively, plus the addition of a soft power law (which could be due to scattering) can describe well the overall shape of the X-ray spectrum of the two sources. Extrapolating the intrinsic hard power law to soft X-rays we find that the unabsorbed value for the X-ray loudness is $\alpha_{\text {ox }} \sim 1.6$ and $\sim 1.5$ in the case of PG $1411+442$ and Mrk 304, respectively. This result is in agreement with the results of Brandt et al. (2000), that absorption is the primary cause of soft X-ray weakness in quasars. However, at the same time, the intrinsic spectral slope of Mrk 304 is flatter than the canonical value of $\Gamma \sim 1.9$. Taking into account the lack of significant iron line emission in this object as well, our results also support the suggestion of Risaliti et al. (2003) that there may be a class of quasars with intrinsically flat X-ray spectra.

We also find strong evidence for the presence of ionized material in the lines of sight. In both objects we detect significant emission at $\sim 0.59 \mathrm{keV}$, consistent within the errors, with the line energies of $\mathrm{O}$ VII. This line emission could originate from diffuse gas with temperatures $k T \sim 0.15-0.3 \mathrm{keV}$, 
which is moving at speeds of a few thousand $\mathrm{km} \mathrm{s}^{-1}$. We also detect significant edges at $\sim 0.7 \mathrm{keV}$, which could be the edge associated with the O VII line emission (although the edge energy is smaller than the energy of the O VII absorption edge). The quality of the present data is not good enough for a detailed physical investigation of the geometry of the absorbing material (a uniform or partially covering screen) and its physical condition (neutral and/or ionized, perhaps material at different states of ionization).

In a way our results demonstrate the similarity in the environment near the central source in Seyfert 1 galaxies and in quasars. Although the majority of the optically selected quasars have column densities less than $\sim 10^{21} \mathrm{~cm}^{-2}$, our results show that there are also quasars with column densities of the order of $10^{22}-10^{23} \mathrm{~cm}^{-2}$. It should be noted that most of the known genuine X-ray weak quasars (i.e., if not classified as BAL QSO) are rather low luminous objects, which suggests that the central energy output affects the accretion geometry - or vice versa. Furthermore, the fact that we also find strong evidence for ionized gas in the line of sight, implies that the "warm absorber" phenomenon may be as common in quasars as it is in Seyfert 1 objects (e.g. George et al. 1998).

Acknowledgements. This work is based on observations with XMM-Newton, an ESA science mission with instruments and contributions directly funded by ESA Member States and the USA (NASA). This research has made use of the NASA/IPAC Extragalactic Data Base (NED) which is operated by the Jet Propulsion Laboratory, California Institute of Technology, under contract with the National Aeronautics and Space Administration. EF acknowledges support by the International Max-Planck Research School on Astrophysics (IMPRS).

\section{References}

Ballantyne, D. R., Vaughan, S., \& Fabian, A. C. 2003, MNRAS, 342, 239

Boroson, T. A., \& Green, R. F. 1992, ApJS, 80, 109

Brandt, W. N., Laor, A., \& Wills, B. J. 2000, ApJ, 528, 637

Brinkmann, W., Yuan, W., \& Siebert, J. 1997, A\&A, 319, 413

Brinkmann, W., Wang, T., Matsuoka, M., \& Yuan, W. 1999, A\&A, 345,43

Brinkmann, W., Branduardy Raymond, G., Grupe, D., \& Ferrero, E. 2003, A\&A, 398, 81
Della Ceca, R., Palumbo, G. G. C., Persic, M., et al. 1990, ApJS, 72, 471

Done, C., Mulchaey, J. S., Mushotzky, R. F., \& Arnaud, K. A. 1992, ApJ, 395, 275

Ehle, M., Breitfellner, M., Dahlem, M., et al. 2001, XMMNewton Users' Handbook, http://xmm.vilspa.esa.es/ xmm_user_support/external/documentation/ uhb_frame.shtml

Elvis, M. 2000, ApJ, 545, 63

Elvis, M., Wilkes, B. J., \& Lockman, F. J. 1989, AJ, 97, 777

George, I. M., Turner, T. J., Netzer, H., et al. 1998, ApJS, 114, 73

Ghizzardi, S., \& Molendi, S. 2001, Proc. of the Conf., New Visions of the X-ray Universe, ESTEC Nov. 2001

Hutchings, J. B., \& Neff, S. G. 1992, AJ, 104, 1

Jang, M., \& Miller, R. H. 1995, ApJ, 452, 582

Kriss, G. A., Krolik, J. H., Otani, C., et al. 1996, ApJ, 467, 629

Lockman, F. J., \& Savage, B. D. 1995, ApJS, 97, 1

Malkan, M. A., Green, R. F., \& Hutchings, J. B. 1987, ApJ, 322, 729

Mathur, S. 1994, ApJ, 431, L75

Mathur, S., Wilkes, B., Elvis, M., \& Fiore, F. 1994, ApJ, 434, 493

Murray, N., Chiang, J., Grossman, S. A., \& Voit, G. M. 1995, ApJ, 451,498

Mushotzky, R. F. 1982, ApJ, 256, 92

Nandra, K., \& Pounds, K. A. 1994, MNRAS, 268, 405

Netzer, H. 1996, ApJ, 473, 781

Netzer, H., Turner, T. G., \& George, I. M. 1998, ApJ, 504, 680

Otani, C., Kii, T., Reynolds, C., et al. 1996, PASJ, 48, 211

Rachen, J. P., Mannheim, K., \& Biermann, P. 1996, A\&A, 310, 371

Reeves, J. N., \& Turner, M. J. L. 2000, MNRAS, 316, 234

Risaliti, G., Elvis, M., Gilli, R., \& Salvati, M. 2003, ApJ, 587, L9

Ross, R. R., \& Fabian, A. C. 1993, MNRAS, 261, 74

Surace, J. A., Sanders, D. B., \& Evans, A. S. 2001, AJ, 122, 2791

Tananbaum, H., Peters, G., Forman, W., et al. 1978, ApJ, 223, 74

Tananbaum, H., Avni, Y., Green, R. F., Schmidt, M., \& Zamorani, G. 1986, ApJ, 305, 57

Vignali, C., Brandt, W. N., \& Schneider, D. P. 2003, AJ, 125, 433

Wang, T., Brinkmann, W., \& Bergeron, J. 1996, A\&A, 309, 81

Wang, T. G., Wang, J. X., Brinkmann, W., \& Matsuoka, M. 1999, ApJ, 519, L35

Wang, T. G., Brinkmann, W., Matsuoka, M., Wang, X. J., \& Yuan, W. 2000, ApJ, 533, 113

Yuan, W., Brinkmann, W., Siebert, J., \& Voges, W. 1998a, A\&A, 330, 108

Yuan, W., Siebert, J., \& Brinkmann, W. 1998b, A\&A, 334, 498

Zdziarski, A., Johnson, W., Done, C., et al. 1995, ApJ, 438, L63 